\title{
Implicit Tandem Organic-Inorganic Hybrid Perovskite Solar Cells Based on Internal Dye Sensitization: Robotized Screening, Synthesis, Device Implementation, and Theoretical Insights
}

\author{
Allan Starkholm, Lars Kloo, and Per H. Svensson* \\ Cite This: J. Am. Chem. Soc. 2020, 142, 18437-18448 \\ Read Online
}

ABSTRACT: Low-dimensional hybrid perovskite materials offer significantly improved stability as well as an extensive compositional space to explore. However, they suffer from poor photovoltaic performance as compared to the $3 \mathrm{D}$ perovskite materials because of poor charge-transport properties. Herein, we present the concept of internal dye-sensitized hybrid perovskite compounds involving five novel low-dimensional perovskite-type materials 1-5 incorporating triarylmethane, phenazinium and near-infrared (NIR) cyanine cationic dyes, respectively. The synthesis characterization and theoretical analysis of these compounds are presented. Theoretical calculations provide

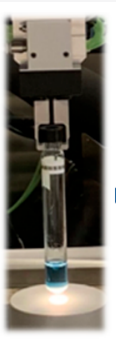

Robotized screening

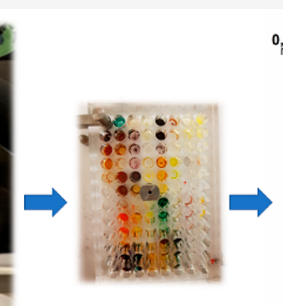

Candidate selection

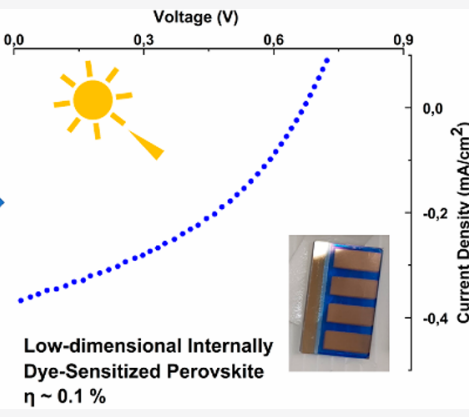
interesting insights into the effects of these dyes on the band structure of the low-dimensional anionic metal-halides and especially highlight compound $\mathbf{1}$ as a promising photovoltaic candidate. Solar cell investigation of devices based on 1 were conducted. The results show an average power conversion efficiency (PCE) of about $0.1 \%$, which is among the highest reported for a $1 \mathrm{D}$ material despite the use of undoped Spiro-OMeTAD as the hole-transport material (HTM). Incident photon-to-electron efficiency (IPCE) spectra confirm the contribution of the dye to the overall photocurrent of the solar cell. Moreover, examination of solar cell devices based on the bismuth-based compound $\mathbf{5}$ resulted in PCEs in the range of $0.1 \%$. This illustrates the potential of this concept to be exploited for lead-free photovoltaics. Finally automated robotized screening of low-dimensional hybrid perovskite materials through the screening robot PROTEUS has emerged as a powerful tool in the search for novel perovskite-like materials. Our work highlights that the use of cationic dyes could induce interesting sensitizing properties to low-dimensional metal-halide chains and may therefore provide inspiration and new design strategies for the synthesis of new lead-free photovoltaic materials

\section{INTRODUCTION}

The past decade has witnessed an intense progress of perovskite solar cells (PSCs), since the very first report of the methylammonium lead triiodide $\left(\mathrm{MAPbI}_{3}\right)$ perovskite in a dye-sensitized solar cell (DSSC). ${ }^{1}$ In a matter of a few years, the power conversion efficiency (PCE) of the 3D perovskite materials was boosted to above $25 \%$ for a single junction cell ${ }^{2}$ and is, as of today, a rich and research-intense field. The hype of the perovskite materials can be traced to its many attractive features, such as efficient light absorption, spectral tunability, high conductivity, ease of fabrication, and potential low production cost. ${ }^{1,3-5}$ Despite the promising results for solar cell applications, there are some aspects retarding large-scale commercialization of perovskite solar cells. These include the intrinsic instability with respect to moist air and the toxicity of lead raising concerns over potential environmental hazards. ${ }^{6,7}$ Consequently, much of the ongoing research today is focused on addressing these challenges. Common approaches to the toxicity issue include partial or complete substitution of lead for tin or bismuth. ${ }^{8}$ Substitution of lead for tin results in a 3D perovskite structure with smaller bandgaps but with poor stability as a major setback because of the facile oxidation of $\mathrm{Sn}(\mathrm{II})$ to $\mathrm{Sn}(\mathrm{IV}) .^{4,9,10}$ Bismuth represents a promising alternative to lead, but the bismuth-based perovskite materials suffer from lower PCEs. ${ }^{11}$ In recent years, the perovskite materials field has seen a development of various $2 \mathrm{D} / 3 \mathrm{D}$ Ruddlesden-Popper perovskite-based devices representing more stable options to the traditional $\mathrm{MAPbI}_{3}$-based PSCs with PCEs reaching levels of the latter ones. ${ }^{12}$ In addition, lowdimensional hybrid perovskite materials are becoming increasingly interesting prospects for photovoltaics because of a vast compositional and structural space with the possibility to incorporate functional organic cations. ${ }^{13,14}$ Numerous 2D, 1D,

Received: June 22, 2020

Published: October 15, 2020 
and OD hybrid perovskite materials have been synthesized and reported in the literature. ${ }^{15}$ Although many of them exhibit interesting properties for photovoltaics, they typically perform poorly as active layers in solar cells as compared to the $3 \mathrm{D}$ perovskite materials. This is mainly attributed to the reduced electronic dimensionality in low-dimensional materials, because the semiconducting inorganic network is not structurally connected in all three dimensions in contrast to $\mathrm{MAPbI}_{3}{ }^{16}{ }^{6}$ Therefore, they suffer from poor charge-transport properties and larger electronic bandgaps. Efforts to solve these issues are made within the perovskite materials community, and some strategies include surface engineering, doping, and incorporation of semiconducting cations. ${ }^{17}$

In our group, we are interested in low-dimensional hybrid perovskite materials and aim to resolve the issues associated with these materials. Previously, we have reported on hybrid perovskite compounds incorporating polyiodide entities as linking units between the inorganic slabs as one strategy to enable a 3D electronic path for photoexcited electrons. ${ }^{18}$ The reported polyiodide systems all show small bandgaps with the polyiodide orbitals overlapping with those of the inorganic metal-halide sheets. A different, but related, approach of resolving the charge-transfer issues is to incorporate functional cations into perovskite-like structures. ${ }^{17}$ Some different strategies have been investigated and reported by different research groups. Functional cations, such as tetrathiafulvalene, oligothiophenes, pyrenes, viologens, and naphthalenediimides are examples that can be found in literature. ${ }^{19-25}$ Moreover, attempts to form charge-transfer complexes between 1D anionic iodoplumbate chains was recently reported with promising light-harvesting properties. ${ }^{26}$ One additional possibility is to include dyes as the cationic species in the perovskite materials. Reports on hybrid perovskites containing lightabsorbing cations are scarce. ${ }^{20-22,25,27,28}$ However, those that exist indicate promising properties for photovoltaic applications, such as NIR absorption to name one of particular interest. $^{27}$ In this work, we will investigate dye-containing hybrid perovskite materials experimentally and theoretically aiming for solar cell applications. Cationic dyes offer a possibility to incorporate organic dyes into a perovskite-like structure with the potential to select and align a complementary light absorption range to the low-dimensional inorganic perovskite network, which typically absorbs light between 400 and $450 \mathrm{~nm}$. Consequently, it is particularly interesting to incorporate cations that absorb light at longer wavelengths of the visible-light spectrum and as such form an implicit tandem perovskite material in which the dye sensitizes the inorganic host network. The solar radiation spectrum also reveals that there is a considerable amount of energy in the infrared (IR), as well as the NIR region, that can be harvested. Hence, cationic dyes absorbing NIR light qualify as promising candidates for light harvesting in photonic devices. ${ }^{29}$ In addition, as the compositional space for low-dimensional organic-inorganic hybrid perovskite materials is vast, the need for fast screening and synthesis of candidate materials is crucial to find suitable photovoltaic materials candidates in a reasonably short time. Robotized screening thus becomes a powerful tool for this purpose. In this work, we present the results of an initial robotized screening approach and report five new low-dimensional hybrid perovskite materials internally sensitized with cationic dyes Victoria Blue B (VBB), phenosafranine (PS), and 3,3'-diethylthiatricarbocyanine (DTTC) (Figure 1). The robotized screening approach
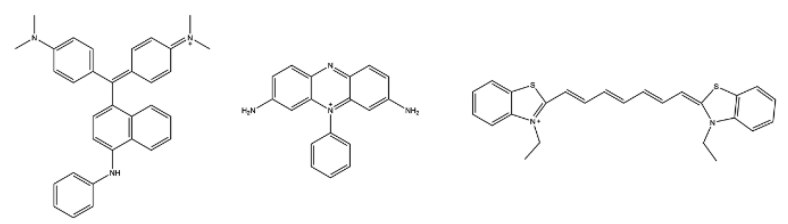

Figure 1. Three cations used in this work, VBB (left), PS (middle), and DTTC (right).

utilizing the PROTEUS robot aims to screen for compositional "islands" of interest, in terms of the formation of new perovskite-like compounds, in the multidimensional and vast compositional space available. The initial screening represents the first step in the process to identify new compounds, after which optimized synthesis and subsequent characterization is performed in the more limited compositional regions ("islands") indicated by the screening (more information regarding the robotized screening is given under the Experimental Section and in the Supporting Information). Furthermore, we have investigated the novel compounds theoretically and experimentally as solar cell materials. The dyes chosen exhibit absorption ranges from the red- to the blue invoking also the NIR spectrum. The new compounds show interesting and promising properties as active layers in perovskite-type solar cells.

\section{RESULTS AND DISCUSSION}

Robotized Screening. The perovskite family of compounds constitutes a wide variety of structural types closely related to the archetypical corner-shared 3D structure. Despite the rapid development of the perovskite field, the emergence of new and efficient alternatives is slow. High-throughput screening (HTS) using automated robotized screening may therefore become an important tool to accelerate the identification of new perovskite materials for optoelectronic applications. Attempts of HTS for perovskites have recently been made, although in small scale and only partially automated. $^{30,31}$ Specifically, in this work, HTS of lowdimensional perovskite-type materials utilizing cationic dyes as "internal sensitizers" for the inorganic perovskite framework has been applied to identify new candidate materials for solar cell application. The PROTEUS robot is designed to intelligently explore the chemical space in the solid-state utilizing a unique combination of liquid dispensing robots, as well as analytical and monitoring equipment, such as turbidity, polarized light intensity (PLI), Raman spectroscopy, and X-ray powder diffraction (XRPD). The target for our screening was to rapidly identify novel crystalline dye-perovskite compounds that would emerge from mixing cationic dye salts with metal halides. Once what compositional combinations to screen is defined, stock solutions of the compounds are prepared in suitable solvents and at relevant concentrations. In this work, cationic dye salts were prepared in DMF in a concentration range of $0.1-0.3 \mathrm{M}$. The screening library of cationic dyes were chosen based on structural type, light absorption range, and commercial availability. Five metal halides $\left(\mathrm{PbI}_{2}, \mathrm{SnI}_{2}\right.$, $\mathrm{BiI}_{3}, \mathrm{SbI}_{3}$, and $\mathrm{ZnI}_{2}$ ) were used in the screening and prepared separately as stock solutions in DMF. Subsequently, the stock solutions were submitted and fed into the robotic system through a computer-controlled system.

Experimental parameters, such as stoichiometry, temperature and crystallization method can be selected and executed through the computer interface to the robotic system. The 
software calculates the amounts required of each component, and the robot will automatically dispense the correct volumes in the liquid handling stations once the screening experiments have been initiated. The reactions in this work were all performed at room temperature, and the screening experiments were designed to mix the cationic dyes with the metal halides in a 1:1, 2:1, and 1:2 stoichiometric ratio. Whether formation of a crystalline precipitation has occurred is determined through the analytical chains defined and monitored using the analytical tools mentioned above. Initial precipitation is detected through turbidity measurements by sensors in the thermal blocks, where the reaction vessels are placed. The formation of crystalline materials is subsequently checked through PLI measurements. If the sample is identified as crystalline, it continues further through the analytical sequence involving Raman spectroscopy and XRPD. In this step, it can be determined whether the crystalline sample represents a previously unknown phase or not. If no precipitation can be verified, the robot continues with next step in the experiment flowchart. Different crystallization techniques can be applied, involving antisolvent addition, evaporation, controlled cooling, or slurry reaction. For the purpose of this work, the antisolvent addition approach was selected as the most suitable method for rapid screening and identification of candidate materials and was therefore applied. After crystalline materials were identified, the robotic cycle ends by sampling specified volumes of the reaction suspension into a 96-well plate for further crystal growth. Pictures of the well-plate positions are taken subsequently with a polarized light microscope. Five novel and interesting compounds were identified, and optimized bulk synthesis and single-crystal growth were performed "manually" for further characterization and solar cell evaluation following the compositional space identified by the robotic screening. The following section describes the identified compounds and the respective crystal structures.

Crystal Structures. Slow diffusion of diethyl ether into a DMF-solution containing VBB chloride, $\mathrm{PbI}_{2}$, and $\mathrm{NaI}$ yielded dark needlelike crystals of $\mathbf{1}$ that crystallize in the monoclinic space group $P 2_{1} / n$ (No. 14), with corresponding CCDC number 2003729. Compound $\mathbf{1}$ is a $1 \mathrm{D}$ hybrid perovskite material consisting of face-sharing $\left[\mathrm{PbI}_{3}\right]_{n}{ }^{-}$chains running along the crystallographic $b$-axis with VBB cations and dimethylformamide (DMF) molecules residing in the voids between the inorganic chains (Figure 2). The VBB cations exhibit a stacking along the $b$-axis, in parallel and between the iodoplumbate chains (Figure 2). Short-distance, edge-to-face, as well as displaced aromatic interactions are observed in the extensive stacking of the iminium cations. The iodoplumbate chains are connected on either side of the VBB cation through C-H---I contacts. The DMF solvent molecules act to stabilize the crystal lattice through $\mathrm{N}-\mathrm{H}---\mathrm{O}$ hydrogen bonding with the primary ammonium moiety, combined with short $\mathrm{C}-\mathrm{H}$ contacts to the iodoplumbate chains. Compound 2 crystallizes in the monoclinic space group $P 2_{1} / n$ (No. 14) as a $1 \mathrm{D}$ hybrid perovskite structure consisting of face-sharing $\left[\mathrm{PbI}_{3}\right]_{n}{ }^{-}$anionic chains together with PS cations and dimethyl sulfoxide (DMSO) molecules (Figure 3). The crystal structure is of poor quality and should be considered a model structure with the main purpose as platform for band structure calculations. The CIF file can be found in the Supporting Information. A close inspection of the structure reveals pairwise T-shaped $\pi$ stacking patterns of the neutral aromatic backbone nitrogen

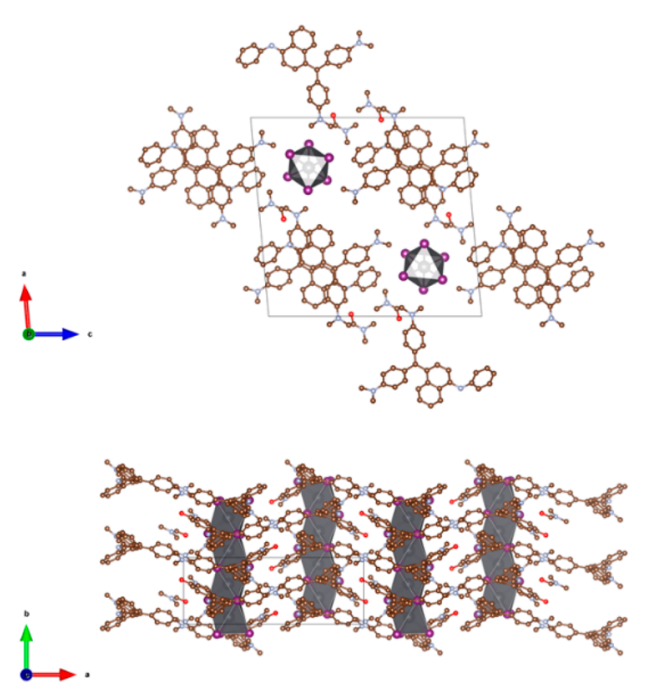

Figure 2. Crystal structure of compound 1 along the crystallographic $b$-axis (upper) and a $2 \times 2 \times 1$ supercell showing the packing of the cations and the extension of the iodoplumbate chains (lower). Hydrogen atoms are omitted for clarity.

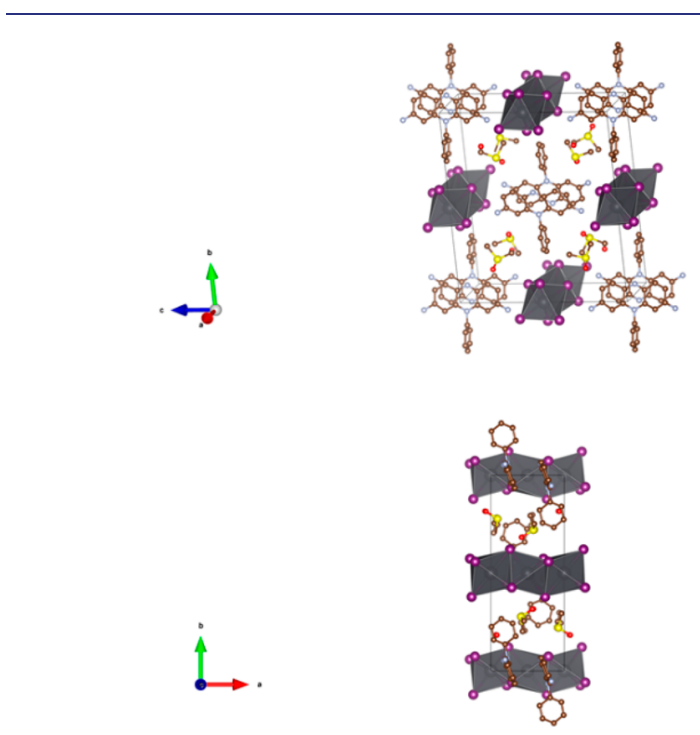

Figure 3. Model of the crystal structure of compound 2 with the unit cell shown along the crystallographic $a$-axis (upper) and the $c$-axis (lower). Hydrogen atoms are omitted for clarity.

atoms with the aromatic side groups of a second cation with a corresponding $\mathrm{C}-\mathrm{H}---\mathrm{N}$ contact of $2.59 \AA$. The third cation along the stacking direction exhibits longer $\mathrm{N}-\mathrm{H}---\mathrm{C}$ contacts with the second PS cation without any signs of $\pi-\pi$ interaction. Instead, it forms the $\mathrm{T}$-shaped aromatic interaction with the next cation. The cations hence form a network with pairwise $\pi-\pi$ stacking motifs along the crystallographic $a$-axis. The outer amino groups of the cation backbone form hydrogen bonds with the oxygen atoms of the DMSO molecules and with the iodides of the iodoplumbate network, respectively. In addition, the cations stack along the $a$-axis, running in parallel with the polymeric iodoplumbate chains. Slow evaporation of a DMF solution containing DTTC iodide and $\mathrm{BiI}_{3}$ in a $2: 1$ ratio yielded crystals of 3 after 2 weeks. Compound 3 (CCDC: 2003730) crystallizes in the triclinic space group $\overline{P 1}$ as a DMF solvate. It represents a lead-free analogue in the perovskite family of compounds and consists of 
binuclear $\left[\mathrm{Bi}_{2} \mathrm{I}_{9}\right]^{2-}$ anions and DTTC cations forming a complex extended structure (Figure 4). The cations are

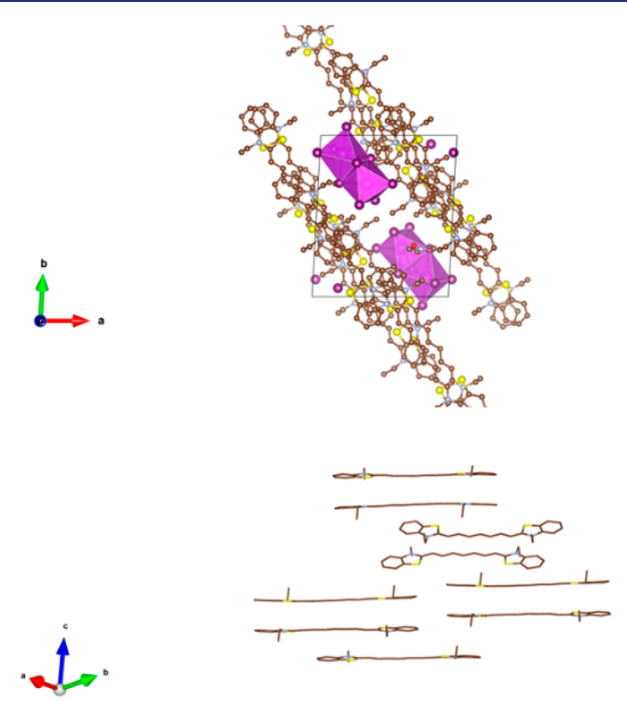

Figure 4. Unit cell of compound 3 along the $c$-axis (upper). The arrangement of the DTTC cations is shown below. Hydrogens are omitted for clarity.

stacked through secondary interactions mediated by the strongly delocalized $\pi$-electrons over the conjugated cyanine backbone, resulting in strong dipole coupling of the cations. The packing of the cyanine cations takes the form as a combination of alternating face-to-face and face-to-end stacking sheets (Figure 4). The face-to-face and face-to-end couplings display short $\mathrm{C}-\mathrm{C}$ contacts within the range of typical aromatic $\pi-\pi$ interactions. The antisolvent diffusion method was used to grow dark, platelike crystals of $\mathbf{4}$ from a DMF solution of DTTC iodide, $\mathrm{PbI}_{2}$, and $\mathrm{NaI}$. The crystal structure of $\mathbf{4}$ is of poor quality and should, just as 2, consequently be regarded as a model structure used as input for the band structure calculations.

Compound 4 crystallizes in the monoclinic space group $P 2_{1} /$ $c$ and contains face-sharing $\left[\mathrm{Pb}_{2} \mathrm{I}_{6}\right]_{n}{ }^{2-}$ chains and DTTC cations (Figure 5). The cations form an extensive stacking network consisting of primarily face-to-end stacks, forming two sets of stacks running in separate directions but interacting through $\mathrm{C}-\mathrm{H}$ contacts. Secondary interactions link the polymethine chains, with the aromatic rings via intermolecular C---C contacts ranging from 3.2 to $3.4 \AA$ combined with aromatic $\pi-\pi$ interactions at $3.38 \AA$, giving rise to an extended staircase stacking (Figure 5), referred to as J-aggregation. This is commonly found among cyanine dyes. ${ }^{32}$ Furthermore, coupling of the cations with the apical iodides of the anionic chains occur through C-H---I contacts of 3.01, 3.07, and 3.13 A.

Crystals of $\mathbf{5}$ grew after two months from a saturated DMFsolution of $\mathrm{VBB}$ chloride, $\mathrm{BiI}_{3}$, and $\mathrm{NaI}$. It crystallizes in the triclinic space group $P 1$ as a DMF-solvate containing VBB cations and square pyramidal mononuclear $\mathrm{BiI}_{5}{ }^{2-}$ anions, as depicted in Figure 6. The crystal structure should be regarded as a model structure, because the crystal data are of rather poor quality. The packing of the aromatic VBB cations differs from that in compound $\mathbf{1}$, as it is dominated by aliphatic $\mathrm{CH}-\pi$ interactions and distorted edge-to-face (T-shaped) interactions with distances in the range of 2.7-2.9 $\AA$. The DMF molecules

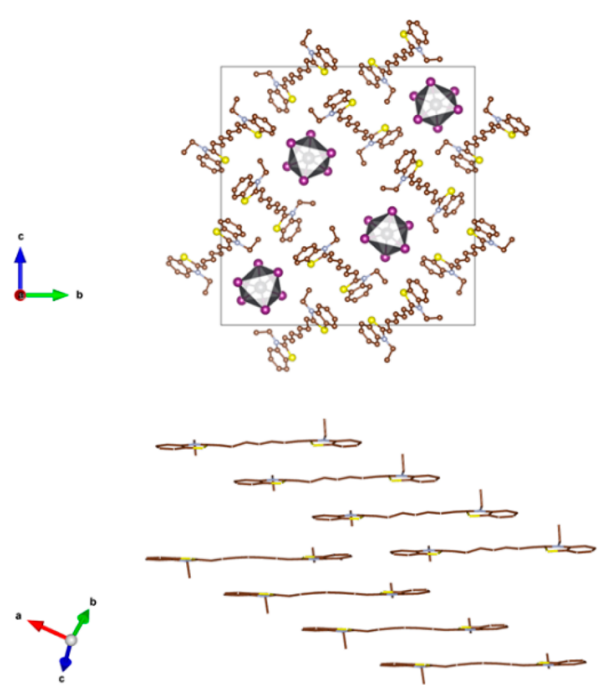

Figure 5. Model of the crystal structure of $\mathbf{4}$ along the $a$-axis (upper) and the staircase arrangement of the cations (lower). Hydrogens are omitted for clarity.

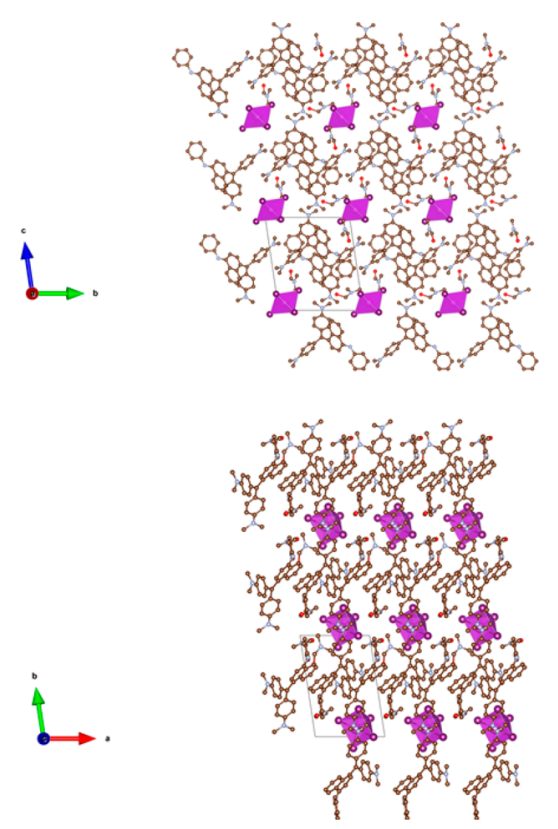

Figure 6. Model of the crystal structure of compound 5 along the crystallographic $a$-axis (upper) and along the $c$-axis (lower).

form hydrogen bonds to $\mathrm{NH}$ groups of the VBB cations and connect these with the $\mathrm{BiI}_{5}{ }^{2-}$ anions through multiple $\mathrm{C}-\mathrm{H}$ contacts. In addition, the crystal structure reveals an anion $-\pi$ interaction at about $2.96 \AA$ between the pentaiodobismuthate and one of the phenyl rings in the triarylmethane backbone of the VBB cation.

For reference, the crystal structure of the iodide salt of VBB was determined, denoted 6 (CCDC: 2003731), and can be regarded as an organic iodide salt structure with a band gap (molecular HOMO-LUMO gap) of about $1.62 \mathrm{eV}$. Equivalently, the calculated bandgap (molecular HOMOLUMO gap) of the previously reported iodide salt of DTTC is $1.85 \mathrm{eV}$.

Band Structure Calculations. Band structure calculations were performed for compounds $\mathbf{1}, \mathbf{2}, \mathbf{3}, \mathbf{4}$, and 5 on the basis of the crystal structures determined. It is considered that the 

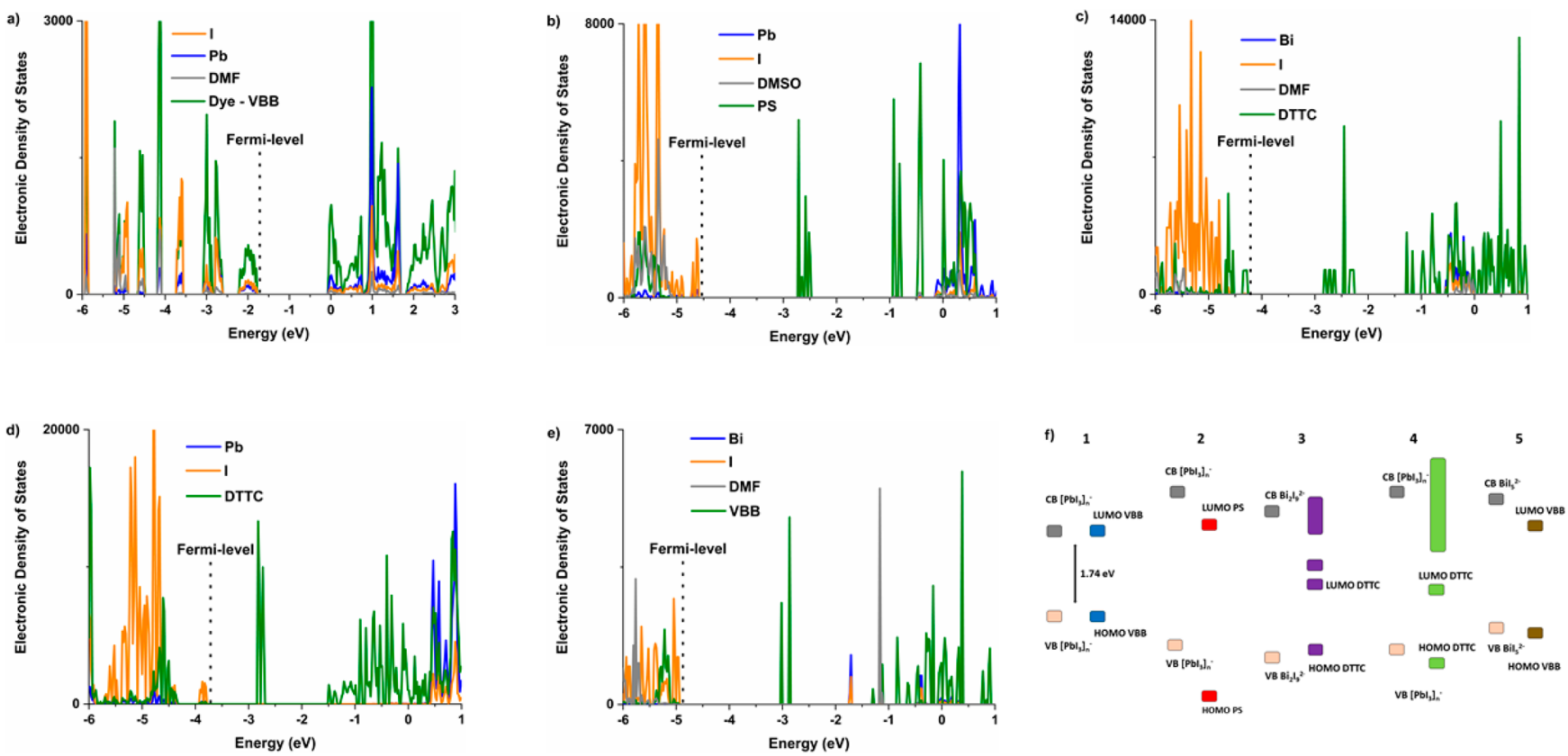

Figure 7. Density of electronic states (DOS) of compound (a) 1, (b) 2, (c) 3, (d) 4, and (e) 5. (f) Comparison of the VB and CB levels of the inorganic chains vs the VB and $\mathrm{CB}$ levels of the cationic dyes in 1, 2, 3, 4, and 5 . The energy levels between the compounds are relative to each other.

model structures of $\mathbf{2}, \mathbf{4}$, and $\mathbf{5}$ are sufficiently well described to constitute a platform for understanding the interaction between the dye cations and the metal-iodide backbone in the structures and thus contribute to our understanding of the concept of internal dye sensitization. To facilitate the following discussions regarding the band structures, we define the HOMO-LUMO energy levels as the highest occupied and lowest occupied energy bands (levels) belonging to the dye cations, and the valence band (VB) and conduction band (CB) refer to the inorganic iodometallate framework. To display how the energy levels of the HOMO and the LUMO of the incorporated dyes interact with the $\mathrm{VB}$ and $\mathrm{CB}$ of the iodometallate host, we used both band structures (Figure S5) and density of states (DOS) to illustrate to what extent the molecular orbitals of the compound building blocks contribute to the bands of primary interest, i.e., those close to the top of the $\mathrm{VB}$ and the bottom of the $\mathrm{CB}$ of the inorganic host structure.

For efficient charge transfer from the dye to the inorganic framework upon photoexcitation, the LUMO energy level of the dye cation needs to be more positive than the $\mathrm{CB}$ of the iodometallate host (the electron vacuum energy level will be used for energy level comparisons throughout this work). This would make electron injection from the dye LUMO to the host $\mathrm{CB}$ feasible and, consequently, the synergy of incorporating complementary light absorption into the low-dimensional perovskite network can be exploited.

The band structure of compound 1 reveals a band gap of approximately $1.74 \mathrm{eV}$. In the electronic DOS it can be observed that the HOMO and LUMO energy levels of the VBB cation are at the same energy levels as the VB and CB of the inorganic perovskite-type network. It is further noted that the dye HOMO-LUMO are well dispersed and integrated with the orbitals constituting the VB and $\mathrm{CB}$ of the $\left[\mathrm{PbI}_{3}\right]_{n}{ }^{-}$ chains (Figure 7a). Inspection of the molecular orbitals shows a contribution of all parts of the compound, the dye cation, the lead- and iodine-orbitals to the bands around the band gap
(Figure 7a). This level of orbital mixing indicates a potential for charge transfer between, or delocalization of, the electrons in the VBB cation and the anionic network.

Analysis of the band structure and DOS of the phenosafranine hybrid perovskite 2 reveals an indirect electronic transition from the $\mathrm{VB}$, primarily dominated by $\mathrm{I}^{-}$ orbitals, to the dye LUMO (Figure $7 \mathrm{~b}$ ). The bandgap of this transition was calculated to $1.88 \mathrm{eV}$. Furthermore, the position of the LUMO energy level of the PS cationic molecule is approximately in the middle of the VB-CB bandgap of the iodoplumbate system. There are additional empty orbital bands belonging to the PS molecules between the LUMO of the dye and the $\mathrm{CB}$ of the iodoplumbate network. The distinct LUMO level of the PS cations and the lack of dispersion in the vicinity of the $\mathrm{CB}$ of the inorganic perovskite-like structure may originate from the absence of a continuous $\pi-\pi$ stacking of the PS cations along their packing direction. As a consequence, the PS cation in $\mathbf{2}$ is expected to be a poor sensitizer for the inorganic host network.

Looking at the DOS of the lead-free hybrid perovskite material, 3 (Figure $7 \mathrm{c}$ ), one can note a resemblance to the DOS of compound 2. However, some clear differences can also be discerned. The HOMO level of the DTTC molecules can be found just below the Fermi level, whereas the VB of the dimeric bismuth anion is slightly more negative than the HOMO level of the dye. The closest empty orbital band belongs to the DTTC cations, and consequently, the bandgap in this material rather corresponds to a HOMO-LUMO transition of the dye. The calculated bandgap for this transition is $1.48 \mathrm{eV}$. In close proximity to the LUMO of the DTTC there are additional empty orbital bands as well as around -0.5 $\mathrm{eV}$, corresponding to the $\mathrm{CB}$ of the inorganic host. Around the $\mathrm{CB}$ of the inorganic entity there are well dispersed bands corresponding to the dye molecules, potentially suggesting a higher probability that electron injection from the dye could take place. The compounds $\mathbf{2}$ and $\mathbf{3}$ are quite analogous with the difference that the dye in 3 possesses "LUMO bands" close 
in energy level, which could be more beneficial for solar cell applications. A possible explanation for the formation of these distinct gaps in the middle of the inorganic VB-CB bandgap in 3 can be found in the stacking of the DTTC cations. We note the formation of a mixture of J-aggregate and $\mathrm{H}$-aggregate type of stacking in $\mathbf{3}$ with solvent molecules interacting and thereby linking different stacks as well. J-aggregation generally results in red-shifted molecular energy levels, whereas $\mathrm{H}$-aggregation typically causes a blue-shift. ${ }^{32}$

For the 1D hybrid perovskite compound 4, the band structure and DOS (Figure 7d) resemble those for 3 but with some differences. Unlike $\mathbf{3}$, the bandgap in 4 corresponds to a transition from the VB of the inorganic entity, dominated by iodine orbitals, to the LUMO of the DTTC cation. This bandgap was calculated to $0.99 \mathrm{eV}$. At slightly more negative energy levels, one can find the major part of the VB with a predominant contribution from the dye HOMO and iodine orbitals and the excitation from this level to the dye LUMO is about $1.5 \mathrm{eV}$. At more positive energy levels than the dye LUMO, there are also empty cation dye bands that are well dispersed around the $\mathrm{CB}$ of the iodoplumbate. In 4, the extensive stacking of the aromatic molecules is exclusively of Jaggregate type, where two different sets of stacking patterns overlap. We speculate that this causes a more dispersive nature of the empty orbital bands belonging to the dye at around the energy levels of the material $\mathrm{CB}$.

Examination of the band structure as well as the DOS (Figure 7e) of the lead-free compound 5 provides interesting insights into the electronic transitions. The band structure calculations result in a direct bandgap of $1.94 \mathrm{eV}$. In contrast to the fully dispersed cationic and anionic bands in $\mathbf{1}$, compound $\mathbf{5}$ displays more separated bands above the Fermi level. The lowest energy excitation occurs from the material $\mathrm{VB}$, which is well dispersed with the VBB HOMO level, to the dye LUMO located at approximately $-3.1 \mathrm{eV}$.

The top of the VB is mainly dominated by iodine orbitals and, at slightly more negative energy levels, both iodine orbitals and the dye HOMO contribute. The $\mathrm{CB}$ of the mononuclear pentaiodobismuthate complex is located at around $-1.75 \mathrm{eV}$.

Moreover, there are other possible scenarios that could occur by incorporating a dye within a perovskite framework. In a perovskite-type of solar cell, the light-absorbing material is typically sandwiched between an electron-transport material (ETM) and an HTM in order to reduce recombination losses and boost the overall conversion efficiency. For electron injection to proceed from the perovskite to the ETM in such a device, the $\mathrm{CB}$ of the perovskite material needs to be more positive than that of the ETM. For a low-dimensional hybrid perovskite containing a sensitizing dye, a case where the LUMO of the cationic dye is more positive than the $\mathrm{CB}$ of the ETM could exist as well. This, in fact, would mimic the function in pure dye-sensitized solar cells, where the dye typically is designed to inject into a suitable ETM (most commonly, $\left.\mathrm{TiO}_{2}\right) .{ }^{33}$ This would indicate a possibility of a photoexcited electron to be injected directly from the dye LUMO into the CB of the ETM, completely bypassing the perovskite-type host in 1-5. To investigate if such a phenomenon contributes significantly to the observed photocurrent in the resulting solar cell devices, reference experiments examining the effect of the organic dye salt VBB iodide were also included in the subsequent section on solar cell performance. In addition, screening of different ETMs would be desirable to study this phenomenon in depth, because the effect of the different energy levels on this process may differ significantly. In general, low-dimensional hybrid perovskite materials display wider bandgaps than the standard 3D ones, ${ }^{17}$ and hence the use of an ETM with a more negative CB, such as tin(IV) oxide, $\mathrm{SnO}_{2}$, could be of interest for sake of comparison. To summarize, the band structures and DOS of the five new compounds reveal interesting features of internal dye-sensitization of low-dimensional hybrid perovskite materials. All five are different in the sense that the relative dye HOMO-LUMO levels within the iodometallate slabs are different, and the degrees of dispersion differ significantly as well. Compound 1, in particular, exhibits a remarkably small bandgap for a 1D structure with respect to the polymeric iodoplumbate anions, where the VBB cation seems to induce a narrowing of the bands in the electronic band structure. The relative $\mathrm{HOMO} / \mathrm{VB}$ and $\mathrm{LUMO} / \mathrm{CB}$ energy levels of the cationic dyes and the iodometallates in the five compounds are illustrated in Figure $7 f$. In conclusion, compound 1 possesses interesting properties as an active layer component for solar cell devices. Careful choice of the various solar device components will be vital to properly align the energy levels of dye-sensitized hybrid perovskite materials to reduce losses because of recombination. The positive results obtained may stimulate further exploration of this novel class of compounds.

Optoelectronic Properties. The optical bandgap of compound 1 was determined by means of diffuse reflectance spectroscopy of a crystalline powder gently pressed onto a surface.

Subsequent transformation of the reflectance data applying the Kubelka-Munk function ${ }^{34}$ yields an indirect bandgap of $1.44 \mathrm{eV}$ (Figure 8a), alternatively $1.55 \mathrm{eV}$ for a direct bandgap
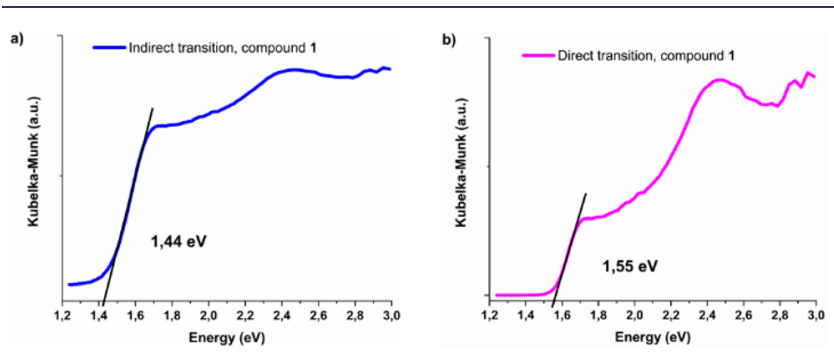

Figure 8. (a) Kubelka-Munk plot for an indirect transition. (b) Kubelka-Munk plot based on a direct transition.

transformation (Figure $8 \mathrm{~b}$ ). It is difficult to clearly assign whether the transition is a direct or an indirect one by examining the Kubelka-Munk functions in Figure 8, as both models yield reasonable results. The band structure of $\mathbf{1}$, however, suggests that the electronic transition might be better assigned as an indirect bandgap. Equivalent reflectance measurements of the dye chloride salt results in an indirect bandgap of $1.36 \mathrm{eV}$, or alternatively, a direct bandgap of 1.52 $\mathrm{eV}$. The experimentally determined bandgap of $\mathbf{1}$ matches the theoretically calculated value of $1.74 \mathrm{eV}$ well, considering typical systematic errors in theoretically estimated bandgaps. It should, however, be kept in mind that the experimental value obtained from the Kubelka-Munk transformation is approximative. To complement the reflectance measurements, we performed absorbance measurements of thin films of 1 (Figure 9b). Absorption is occurring at both shorter and longer wavelengths, between 350 and $400 \mathrm{~nm}$ and a broad absorption between 500 and $700 \mathrm{~nm}$ with an absorption peak at 

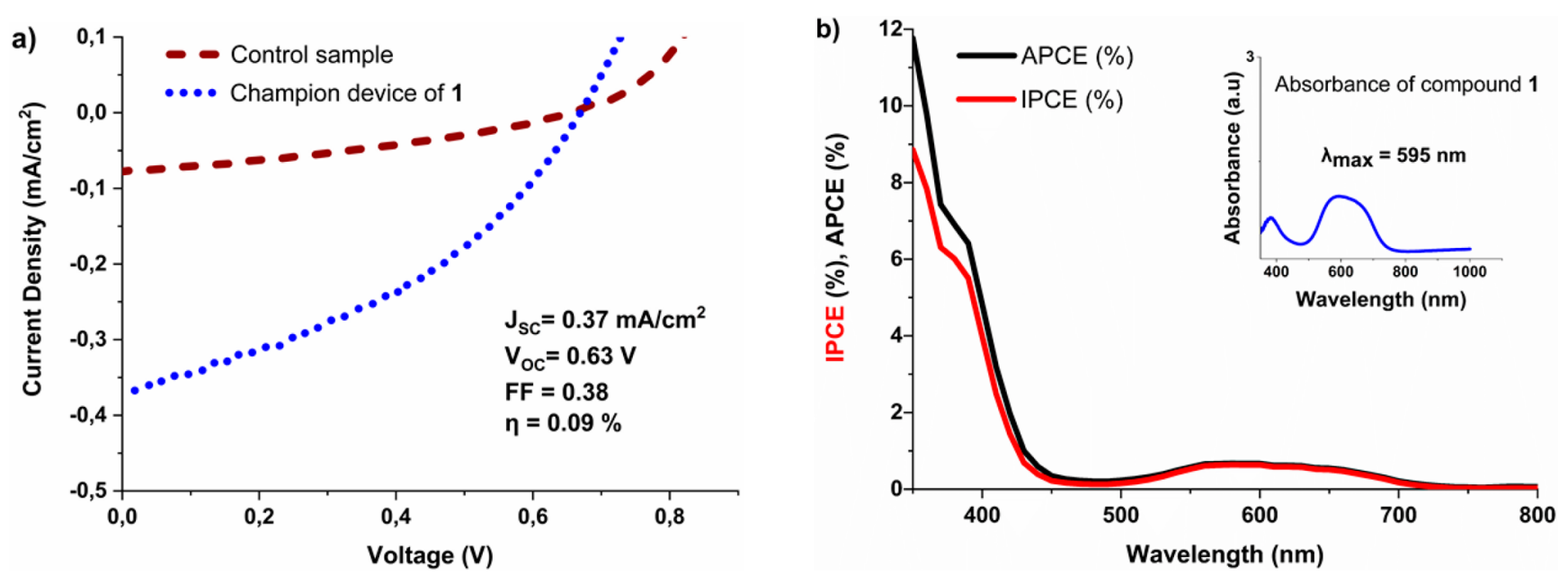

Figure 9. (a) $I V$ curve of the champion device of 1 (dotted line) and the control device of the cationic dye salt, VBB chloride (dashed line). (b) IPCE and APCE spectra of a typical device of $\mathbf{1}$ and the inset illustrates the normalized absorbance of a thin film of $\mathbf{1}$.

approximately $595 \mathrm{~nm}$, in line with previously reported absorption spectra of the dye molecule. ${ }^{35}$ The short-wavelength absorption features can be ascribed to a combination of the anionic $\left[\mathrm{PbI}_{3}\right]_{n}{ }^{-}$chain contribution and a $\pi \rightarrow \pi^{*}$ transition of the aromatic parts in the cation. ${ }^{36,37}$ In summary, the obtained results for compound $\mathbf{1}$ clearly indicate suitably for solar energy harvesting. The Kubelka-Munk absorption spectra of 2 (Figure S4) exhibit an absorption range of 350$600 \mathrm{~nm}$. Extrapolation results in an estimated bandgap of 1.90 $\mathrm{eV}$, consistent with the red color of 2. Examining the KubelkaMunk plot of 3 (Figure S4), one can note a wide absorption range, extending into the NIR region with an indirect bandgap of approximately $1.25 \mathrm{eV}$. Equivalent reflectance measurements of $\mathbf{4}$ (Figure S4) yield similar results as $\mathbf{3}$, an absorption stretching well into the NIR region with a bandgap around $1.30 \mathrm{eV}$. In contrast to 3 , compound 4 lacks significant absorption in the region between 470 and $620 \mathrm{~nm}(2-2.6 \mathrm{eV})$. In 3 , the iodobismuthate anion absorbs strongly in this region whereas the iodoplumbate absorbs light at shorter wavelengths. The Kubelka-Munk plots of compound $\mathbf{5}$ are displayed in Figure S4 and yield an indirect bandgap of $1.52 \mathrm{eV}$ and a direct bandgap of $1.63 \mathrm{eV}$, which are in good agreement with the theoretically calculated values. In conclusion, all five compounds exhibit promising optoelectronic properties as potential light-harvesting materials for solar cell applications.

Solar Cell Application. The above compounds were investigated in a perovskite type of solar cell in order to elucidate its suitability as light-harvesting materials. Compound 2 shows good film forming properties; however, the band structure and the absorption range of the other compounds are more suitable for application in photovoltaic devices. Compounds 3 and $\mathbf{4}$ are both highly interesting candidate materials considering the NIR absorption. However, the application of $\mathbf{3}$ and $\mathbf{4}$ is deemed to be challenging because of the low solubility of the cation even in highly polar solvents. The hybrid perovskite compound $\mathbf{1}$ was for the above reasons selected as the most promising candidate with respect to the combined optoelectronic properties, solubility and availability. A mesoporous device architecture was employed with the structure $\mathrm{FTO} / c p-\mathrm{TiO}_{2} / m p-\mathrm{TiO}_{2} /$ compound $\mathbf{1} /$ Spiro-OMeTAD/Au. A 0.1 M solution of VBB chloride, $\mathrm{PbI}_{2}$, and $\mathrm{NaI}$ (1 equiv.) was prepared in DMF and stirred at room temperature for $1 \mathrm{~h}$ prior to spin-coating application. Excess $\mathrm{NaI}$ was added to ensure sufficient iodide concentration in the solution to prevent partial chloride substitution, which has been shown to decrease the light-absorption ability of the resulting material. ${ }^{38}$ All deposition steps were performed in air at ambient conditions. A solar simulator illumination of AM1.5 100 $\mathrm{mW} / \mathrm{cm}^{2}$ was applied when evaluating the solar cell performance. The best performing devices demonstrated on average a current density, $J_{\text {sc }}$ of $0.32 \mathrm{~mA} / \mathrm{cm}^{2}$; open circuit voltage, $V_{\text {oc }}$ of $0.65 \mathrm{~V}$; fill factor, $\mathrm{FF}$, of 0.37 ; and power conversion efficiency, PCE, of $0.077 \%$. The champion device showed a $V_{\text {oc }}$ of $0.63 \mathrm{~V}$, a $J_{\mathrm{sc}}$ of $0.37 \mathrm{~mA} / \mathrm{cm}^{2}$, an FF of 0.38 , and a PCE of $\sim 0.09 \%$ (Figure 9a). To the best of our knowledge, these results are among the highest reported for a $1 \mathrm{D}$ hybrid perovskite material. In addition, it should be emphasized that all devices investigated were based on nondoped Spiro$\mathrm{OMeTAD}$ as HTM. The reason is that the doping components normally used for Spiro-OMeTAD are incompatible with the thin films of 1 , because these are damaged upon spin-coating of the HTM solution in the presence of the doping components. To gain further insight into the charge-transport characteristics of the assembled devices, electrochemical impedance spectroscopy (EIS) measurements were performed under illumination. The Nyquist plots (Figure S8) and the equivalent circuit used for modeling the results reveal two EIS semicircles that can be ascribed to the series resistance, $R_{s}$, the recombination resistance, $R_{\text {rec }}$ and the resistance from the HTM, $R_{\mathrm{HTM}}$, following typical models applied to interpret EIS response from perovskite solar cells. The extracted series resistance $\left(R_{\mathrm{s}}\right.$ $=11 \Omega)$ is intermediate and in line with reported values for perovskite solar cells. ${ }^{39,40}$ The recombination resistance obtained $\left(R_{\mathrm{rec}}=7 \mathrm{k} \Omega\right)$ is low in comparison to perovskite solar cells based on $\mathrm{MAPbI}_{3}(\sim \mathrm{M} \Omega)$, and combined with the high $R_{\mathrm{HTM}}(68 \mathrm{k} \Omega)$ there are strong indications that the devices studied in this work suffer from significant recombination losses. These can most likely be attributed to the use of nondoped Spiro-OMeTAD, which acts both as a bad holescavenger for the perovskite film, thus rendering high recombination rates, and serves as an inefficient hole conductor. In addition, it has been shown that doping significantly reduces the charge transport resistance owing to Spiro-OMeTAD while the series resistance remains relatively unaffected. ${ }^{41} \mathrm{~A}$ prediction of the photovoltaic performance when minimizing recombination losses can be found in Figure 

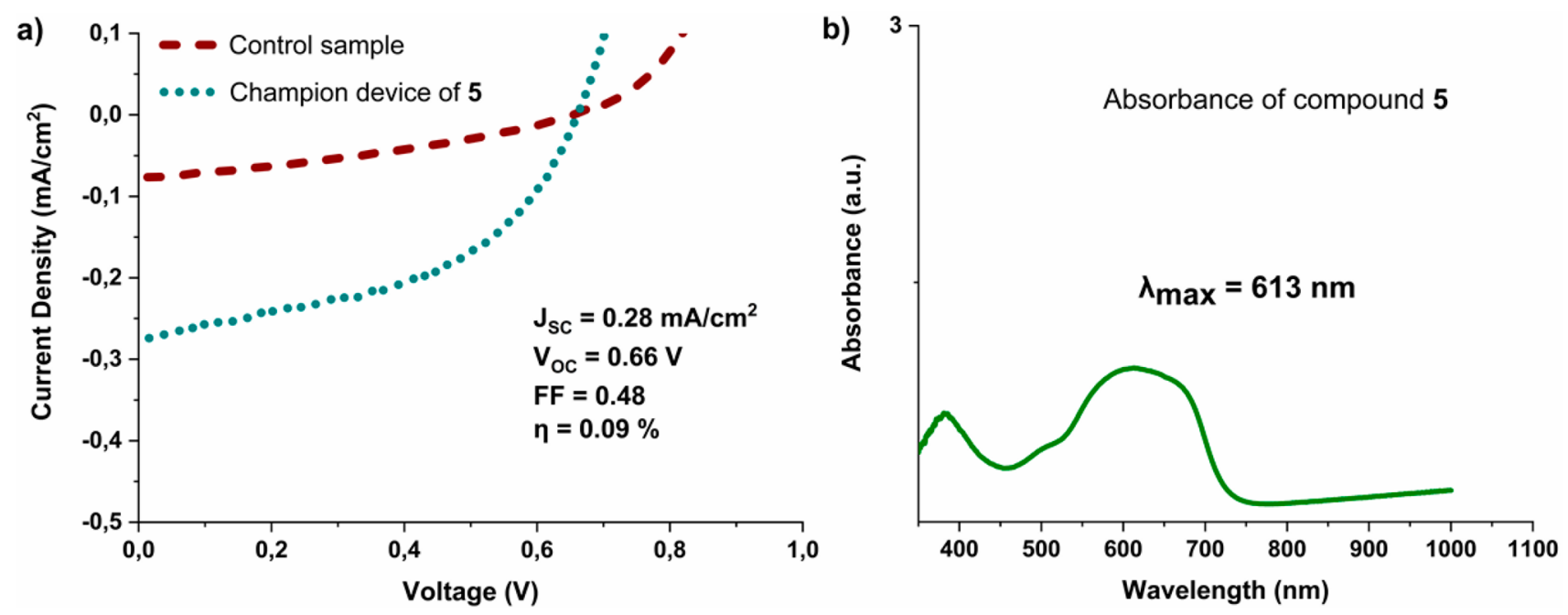

Figure 10. (a) Champion device of a mixture of compound $\mathbf{5}$ (dotted line) and the control device of the cationic dye salt, VBB chloride (dashed line). (b) Normalized absorbance spectra of a thin film of $\mathbf{5}$.

S9. Hence, the results achieved with nondoped Spiro$\mathrm{OMeTAD}$ are very encouraging, since the hole conductivity without doping has been shown to be up to 100 times lower, and doping typically plays a key role in the construction of high-performing perovskite solar cells based on 3D $\mathrm{MAPbI}_{3}{ }^{42,43}$ Spiro-OMeTAD doping has been shown to alter the energy levels of the HTM, which consequently leads to an improved hole mobility and conductivity, ${ }^{44}$ thereby improving the photovoltaic performance.

This in combination with the low concentration necessary for spin-coating of the current precursor solution suggests a substantial potential for improvement of the performance of 1D dye-sensitized hybrid perovskite materials in photovoltaic devices. Furthermore, replacing NaI with HI results in slightly higher currents. This may suggest a different arrangement of the material in the mesoporous $\mathrm{TiO}_{2}$ substrate. The amphoteric nature of $\mathrm{TiO}_{2}$ makes it sensitive to the proton activity of the solutions used for spin coating. Acidic conditions would protonate the $\mathrm{TiO}_{2}$, resulting in a positive net charge of the substrate. Thus, initial alignment of the anionic species, that is, the iodoplumbate chains, seems a logical consequence. Further investigation concerning this phenomenon will be undertaken.

The thickness levels of the active layer (i.e., concentration of the precursor solution) and the ETM play a major role in the resulting performance of the solar cell devices, ${ }^{45,46}$ especially for low-dimensional materials, which generally show lower conductivity as compared to $3 \mathrm{D}$ perovskite materials. ${ }^{16}$ In addition, there are plentiful possibilities for optimization of this system with respect to the parameters addressed, as well as investigating other ETMs and HTMs combined with alternative device architectures. These further steps of development are, however, not within the scope of this work aiming to highlight the conceptual feasibility of internal dye sensitization of low-dimensional hybrid perovskite materials. Control devices of the Victoria Blue B itself as absorber material were fabricated and investigated as a reference, alluding to the discussion in the preceding sections, to detect if the dye, like in a dye-sensitized solar cell of more conventional type, could generate similar photovoltaic effects. Those devices had the structure $\mathrm{FTO} / c p-\mathrm{TiO}_{2} / m p-\mathrm{TiO}_{2} / \mathrm{VBB}$ chloride/ Spiro-OMeTAD/Au. The results indicate a contribution of the dye itself, albeit low (Figure 9a). The $J_{\mathrm{sc}}, V_{\text {oc }}$ and PCE were $0.065 \mathrm{~mA} / \mathrm{cm}^{2}, 0.54 \mathrm{~V}$, and $0.012 \%$, respectively, where the photocurrent density recorded is significantly lower as compared to the dye-sensitized 1D hybrid perovskite material. IPCE spectra were recorded of a statistically averageperforming device of compound $\mathbf{1}$ to display the wavelength dependence of the dye-sensitized $1 \mathrm{D}$ perovskite material (Figure 9b). Inspection of the photocurrent reveals generation of photocurrent over almost the entire visible-light region, with the highest IPCE between 350 and $425 \mathrm{~nm}$ followed by a contribution between 540 and $620 \mathrm{~nm}$, these observations match the range of absorption of compound 1 well and is consistent with the DOS of compound 1. The high contribution at the short wavelength agrees well with previously reported IPCE spectra for $1 \mathrm{D}$ and $2 \mathrm{D}$ perovskite materials with large bandgaps. ${ }^{16,47}$ The obtained IPCE spectra provide a good indication that the VBB dye contributes significantly to the photocurrent of the hybrid perovskite material, although the major contribution is found at shorter wavelengths. Accounting for the absorption range of the dye, we note a photocurrent contribution of $\sim 20 \%$ which highlights the internal sensitization as a potentially viable method to combine the merits of a wide-bandgap semiconductor and a sensitizing dye in a single perovskite-like material. As outlined previously, for efficient charge transfer from the dye to the inorganic network to take place, the LUMO energy level of the dye cation needs to be slightly more positive than the $\mathrm{CB}$ of the iodometallate chains.

Solar cell devices based on the bismuth-based compound 5 containing the VBB cation were also fabricated and evaluated in an equivalent manner as for 1 . The main objective of this comparison is to assess the feasibility of applying a lead-free alternative in solar cells. In this context, it should be highlighted that the inclusion of more components than the mere halidometallates in this family of compounds will extend the available compositional and structural space, and thus the addition of sensitizing dye molecules may constitute an important vehicle to generate lead-free perovskite-like materials showing excellent photovoltaic properties. Similarly to the lead-based system $\mathbf{1}$, bismuth iodide was mixed with VBB chloride and $\mathrm{NaI}$ in DMF in $0.1 \mathrm{M}$ concentration to form a precursor solution. Spin-coating of the precursor solution 
resulted in a greenish-blue, thin film. The absorption spectrum of such a film is shown in Figure $10 \mathrm{~b}$. We note that the absorption spectrum when replacing $\mathrm{PbI}_{2}$ with $\mathrm{BiI}_{3}$ looks quite similar but with an additional absorption feature around 500 $\mathrm{nm}$ corresponding to an iodobismuthate entity.

This implies that the obtained compound covers a larger part of the visible-light spectrum. Subsequent investigation of these devices, with the same mesoporous device architecture and conditions as used for compound $\mathbf{1}$, yielded results akin to the lead-based devices but with much better fill factors (Figure 10a). The photovoltaic performance data of the investigated compounds are summarized in Table S2. The fact that the fill factors are significantly higher when using bismuth iodide implies that these devices may suffer from less recombination. This could, in turn, mean that the energy levels of the components are better matched. The champion device using bismuth iodide in the precursor solution generated a device with a current density of $0.28 \mathrm{~mA} / \mathrm{cm}^{2}$, an open-circuit voltage of $0.66 \mathrm{~V}$, a fill factor of 0.48 , and a PCE of $\sim 0.09 \%$. Again, it should be stressed that the measurements were conducted using dopant-free Spiro-OMeTAD as HTM, which indicates a significant potential for improvement. In analogy to compound $\mathbf{1}$, EIS measurements on devices of 5 were performed. Using the same equivalent circuit model (Figure S8) as for devices containing 1 results in a recombination resistance slightly higher than for devices based on $1\left(R_{\text {rec }}=59 \mathrm{k} \Omega\right)$, as well as a lower hole-transport resistance $\left(R_{\mathrm{HTM}}=18 \mathrm{k} \Omega\right)$, possibly reflecting the better fill factors in solar cells based on this material. A possible explanation for the relatively good performance of devices based on $\mathbf{5}$ could potentially be found in the band structure of $\mathbf{5}$, which indicates a direct bandgap. It is, however, difficult to unambiguously assign the electronic transition as a direct one because of the relatively flat bands. Single-crystal X-ray diffraction studies reveal that $\mathbf{5}$ is a OD-mononuclear iodobismuthate, which in turn suggests that the dye-sensitizing approach may also work well for isolated complexes of halidometallates. This could potentially pave way for other mononuclear anions to be explored as lightharvesting materials, in turn opening up an even broader class of compounds to be investigated. More specifically, OD mononuclear anions displaying short intermolecular contacts combined with internal dye-sensitization would be particularly interesting for optoelectronic applications. Recent studies reported perovskite solar cell devices based on the mononuclear $0 \mathrm{D} \mathrm{SbBr}_{6}{ }^{-}$and $\mathrm{TiBr}_{6}{ }^{-}$anions with PCEs up to 3.2 and $3.3 \%$, respectively, providing promising indications of the potential of mononuclear materials. ${ }^{48,49}$ Further investigation is ongoing regarding the bismuth-based system $\mathbf{5}$, as well as with other lead-free alternatives to obtain a better understanding of the structure-property relationships of these internal dye-sensitized low-dimensional systems.

\section{CONCLUSIONS}

Our work has resulted in conceptually new, internally sensitized low-dimensional hybrid perovskite materials 1-5 obtained by using different types of cationic dyes. Subsequent characterization of the optoelectronic properties combined with solar cell application have shown that the concept of internally sensitizing a metal halide with a suitable cationic dye can qualify as a promising future strategy to significantly improve the performance of low-dimensional hybrid perovskite materials and can be utilized as a vehicle to replace lead in such materials. Band structure calculations were conducted to gain insights into the interaction of the cationic dyes with the metalloiodide hosts investigated in this work. Compound $\mathbf{1}$ was shown to possess interesting properties, such as a narrow bandgap with highly disperse bands, as well as a high degree of orbital mixing between the $\mathrm{VBB}$ cation and the $\mathrm{Pb}$ and $\mathrm{I}$ orbitals. Solar cell investigations revealed that internal sensitization indeed contribute to the photocurrent resulting in devices with relatively good photovoltaic performance. In addition, it was shown that the same dye cation, as used in 3 and 4 , can be applied in combination with another metal halide. Furthermore, we have illustrated that an automated robotized screening can be exploited to synthesize lowdimensional hybrid perovskite materials and could hence become an efficient tool for screening the large compositional and structural space opening up when introducing internally dye-sensitizing dyes to perovskite-like materials.

\section{EXPERIMENTAL SECTION}

Robotized Screening. The screening was applied in the search for new dye-containing perovskite materials using the fully automated crystallization robot PROTEUS, which can perform several hundreds of reactions under controlled conditions. This robot is ultimately designed to explore the solid-state space of materials and possesses a unique setup of characterization instrumentation, such as turbidity analyzer, polarized light intensity (PLI), Raman spectrometer, and Xray powder diffractometer (XPRD), aiming to identify new crystalline phases in the compositional space investigated. In addition, sampling of the reaction solutions to a 96-well plate is performed as the final step for growing single crystals. Specifically, in this work, the screening procedure was designed to produce and identify novel dye-sensitized hybrid perovskite materials upon mixing of stock solutions of cationic dyes and metal halides, followed either by solvent evaporation or subsequent antisolvent addition. The cationic dyes and metal-halide solutions were prepared in the range $0.1-0.3 \mathrm{M}$ in DMF or DMSO solvent. The experimental setup is designed to mix the stock solutions in different stoichiometric ratios, after which the reaction vessels are stirred at room temperature for a specified time before continuing the screening cycle. Screening was performed based on three different stoichiometric ratios, 1:1, 2:1 and finally $1: 2$ with respect to the cationic dyes vs metal halide. As outlined in the main text, if no crystalline material was identified upon mixing the dye solutions with the solutions of metal halide, the reaction vessels will proceed to a process stage involving injection of an antisolvent. In this study, isopropanol or toluene were used as antisolvents. Finally, small "islands" in the vast compositional space are identified in the form of crystals formed in specific areas in the well-plate positions, as well as precipitates in the reaction vessels. Thereafter, further optimization work with respect to growth of single crystals suitable for structure determination, or synthesis of larger amounts of material for characterization, is performed on the basis of the compositional guidelines provided by the robotized screening. More detailed information regarding the robotized screening procedure and singlecrystal growth can be found in the Supporting Information where video links illustrating the PROTEUS function can also be accessed.

Device Fabrication. An FTO glass substrate was cleaned prior to layer deposition using acetone, ethanol (99.9\%), and subsequently deionized water under sonication for $40 \mathrm{~min}$ each. A $\mathrm{TiO}_{2}$ blocking layer (Ti-Nanoxide BL/SC, Solaronix) was spin-coated at a speed of $5000 \mathrm{rpm}$ for $30 \mathrm{~s}$, and then baked in an oven at $500{ }^{\circ} \mathrm{C}$ for $1 \mathrm{~h}$ to yield a compact layer of $50-70 \mathrm{~nm}$ thickness. A mesoporous layer of $\mathrm{TiO}_{2}$ was then deposited by spin-coating a solution made by diluting a $\mathrm{TiO}_{2}$-paste (30 NR-D, Dyesol) with ethanol (99.9\%) in a 1:5.5 weight ratio. The spin-coating was performed at $6000 \mathrm{rpm}$ for $30 \mathrm{~s}$, and the substrates were thereafter annealed at $80{ }^{\circ} \mathrm{C}$ for $5 \mathrm{~min}$ before being baked in an oven at $475{ }^{\circ} \mathrm{C}$ for $30 \mathrm{~min}$. The active layer precursor solution was prepared by dissolving VBB chloride $(0.253 \mathrm{~g}$, 1 equiv.), $\mathrm{PbI}_{2}$ (0.23 g, 1 equiv.) and $\mathrm{NaI}(0.0749 \mathrm{~g}, 1.5$ equiv.) in $5 \mathrm{~mL}$ of DMF. After the vial was left to stir $1 \mathrm{~h}$ at room temperature, $100 \mu \mathrm{L}$ of 
the solution was spin-coated at $4000 \mathrm{rpm}$ for $30 \mathrm{~s}$. No postannealing of the resulting substrates was performed. A hole-transport layer (Spiro-OMeTAD, $20 \mathrm{mg}$ in $1 \mathrm{~mL}$ of chlorobenzene) was subsequently spin-coated at $3000 \mathrm{rpm}$ for $30 \mathrm{~s}$. Finally, gold was thermally evaporated at low pressure $\left(1 \times 10^{-6} \mathrm{mbar}\right)$ onto the substrates to produce a counterelectrode layer of $80 \mathrm{~nm}$ thickness. The resulting solar cell working area was $0.126 \mathrm{~cm}^{2}$.

Solar Cell Characterization. The solar simulator used was of model AM 1.5G solar simulator (Newport 91160-1000), whereby an illumination with an incident light intensity of $100 \mathrm{~mW} / \mathrm{cm}^{2}$ was applied as calibrated with a certified silicon solar cell (Fraunhofer ISE). A linear sweep voltammetry (LSV) scan between $1 \mathrm{~V}$ to $-1 \mathrm{~V}$ was performed using a Keithley 2400 electrometer to obtain the device current-voltage $(J-V)$ characteristics.

Incident Photon-to-Current Efficiency Spectra. IPCE spectra were recorded on a computer-controlled setup with a light source ASB-XE-175, a monochromator (Spectral Products CM110), and a Keithley multimeter (model 2700). Calibration was based on a certified silicon solar cell (Fraunhofer ISE).

Electrochemical Impedance Spectroscopy. To evaluate resistances associated with charge-transport, we performed electrochemical impedance spectroscopy (EIS) measurements under illumination and with an applied bias voltage of $50 \mathrm{mV}$ lower than the $V_{\mathrm{OC}}$ using an Autolab PGstat12 potentiostat with an impedance module under a single sine function. The measurements were carried out in the frequency range $0.1 \mathrm{~Hz}$ to $1 \mathrm{MHz}$ with a $10 \mathrm{mV}$ oscillating amplitude.

Optical Spectroscopy. Both absorption and diffuse reflectance spectroscopic measurements on thin films and polycrystalline powders, respectively, were performed at $298 \mathrm{~K}$. An Avantes AvaSpec-2048 dual UV-vis spectrophotometer provided with an integrating sphere with an integrated light source was used to record the optical properties over the spectral range $300-1100 \mathrm{~nm}$. The Kubelka-Munk function, ${ }^{34} k / s=(1-R)^{2} / 2 R$, was used to convert the reflectance data to absorption data and thus to estimate the optical bandgap of the materials studied. Extrapolation of the function to $k=$ 0 , yields an approximate optical band gap value $E_{\mathrm{g}}$.

Single-Crystal X-ray Crystallography. The single crystals of the respective compounds were selected directly from the reaction solution. These crystals were mounted on a cryoloop using Paratone oil. For compounds 1, 3, 5, and 6, a Bruker APEXII diffractometer (Mo $\mathrm{K} \alpha$ radiation), equipped with a $\mathrm{CCD}$ detector, was used to collect single-crystal X-ray data. $\omega$-scans and $\phi$-scans were used to record the data sets, which subsequently were integrated with the Bruker SAINT ${ }^{50}$ software package. Absorption correction (Bruker SADABS $)^{50}$ was based on the fitting of a function to the empirical transmission surface, as sampled by multiple equivalent measurements. Solution and refinement of the crystal structures were carried out using SHELXS and SHELXL within the Bruker program package. ${ }^{58}$ Structure solution by direct methods resolved positions of all atoms except hydrogens. Hydrogen atoms were placed at calculated positions. The crystal structures of $\mathbf{1}$ and 3 contain solvent accessible voids. A summary of pertinent information relating to unit cell parameters, data collection, and refinement is provided in the Supporting Information. As noted in the main text, systems as the ones included in this work show a strong tendency to grow as very small crystals, too small for standard in-house X-ray diffraction. Therefore, synchrotron-based diffraction was necessary for some of the compounds studied. The single-crystal X-ray data for compound 2 were collected at beamline ID29 $(\lambda=0.72931)$, ESRF in Grenoble, France whereas the data for compound 4 were collected at the 311BioMAX beamline $(\lambda=0.68880)$, MAXIV in Lund, Sweden.

X-ray Powder Diffraction. Fresh crystals were picked from the reaction solutions and gently pressed and smeared onto zero background holders ( $\mathrm{ZBHs}$ ). These were mounted in stainless steel holders and then placed in the powder diffractometer. A PANalytical $\mathrm{X}$ 'Pert PRO diffractometer, equipped with a $\mathrm{Cu}$, long fine focus, $\mathrm{X}$-ray tube, and a PIXcel detector were used for the data accumulation, which were performed at room temperature. Automatic divergence and antiscatter slits were used together with $0.02 \mathrm{rad}$ Soller slits and a
$\mathrm{Ni}$ filter. The scan lengths applied were approximately $17 \mathrm{~min}$. For an increase in the randomness of the crystal sample orientations, the samples were spun during the analysis. The samples were analyzed between 2 and $80^{\circ} 2 \theta$ using 255 detector channels.

Band Structure Calculations. Band structures and DOS were calculated using the hybrid B3LYP or cam-B3LYP functionals in the Crystal 17 package. ${ }^{51}$ The basis sets of $\mathrm{I}$ and $\mathrm{Pb}$ were of small-core ECP quality (MDF28 for I and MDF60 for Pb). ${ }^{52,53}$ The valence space was of double- $\zeta$ quality. ${ }^{54,55}$ In these models employing periodic boundary conditions, dye molecule cations were explicitly included at their crystallographic positions. The basis set of $S$ was also of Stuttgart-Cologne ECP type (MWB10) with an associated small and contracted valence space. ${ }^{56}$ The basis sets used for $\mathrm{C}$ and $\mathrm{H}$ were of 3$21 \mathrm{G}$ or 6-31G quality. Band structures were constructed from the web facility, CRYSPLOT. ${ }^{57}$

The dye molecules were studied using both Crystal 17 and the program package Gaussian 16 (rev. B.01). ${ }^{58}$

\section{ASSOCIATED CONTENT}

\section{Supporting Information}

The Supporting Information is available free of charge at https://pubs.acs.org/doi/10.1021/jacs.0c06698.

Description of single-crystal growth procedures for compounds 1-6; tables including structural data of compounds 1, 3, and 6; XPRD patterns of 1 and 5; Kubelka-Munk plots of 2, 3, 4, and 5; band structure graphs of 1-6; schematic illustration and description of the screening robot PROTEUS and the screening workflow; video links illustrating the robot; table of photovoltaic performance of the systems investigated; Nyquist plots from the EIS measurements of $\mathbf{1}$ and $\mathbf{5}$ together with the equivalent circuit models used for fitting; predicted $I V$ curves with minimized recombination losses (PDF)

Crystallographic information files (ZIP)

\section{AUTHOR INFORMATION}

\section{Corresponding Author}

Per H. Svensson - RISE Chemical Process and Pharmaceutical Development, Södertälje 15136, Sweden; Applied Physical Chemistry, Department of Chemistry, KTH Royal Institute of Technology, Stockholm SE-100 44, Sweden; ○orcid.org/ 0000-0003-2410-7366; Email: per.h.svensson@ri.se

\section{Authors}

Allan Starkholm - RISE Chemical Process and Pharmaceutical Development, Södertälje 15136, Sweden; Applied Physical Chemistry, Department of Chemistry, KTH Royal Institute of Technology, Stockholm SE-100 44, Sweden; O orcid.org/ 0000-0002-2032-1966

Lars Kloo - Applied Physical Chemistry, Department of Chemistry, KTH Royal Institute of Technology, Stockholm SE100 44, Sweden; (i) orcid.org/0000-0002-0168-2942

Complete contact information is available at: https://pubs.acs.org/10.1021/jacs.0c06698

\section{Author Contributions}

The manuscript was written through contributions of all authors. All authors have given approval to the final version of the manuscript.

\section{Notes}

The authors declare no competing financial interest. 


\section{ACKNOWLEDGMENTS}

This work was supported by the Swedish Foundation for Strategic Research (SSF) with Grant FID15-0023, ÅForsk foundation under Grant 17-594, the Swedish Energy Agency (Grant ID: 46379-1), and the Swedish Research Council (ID: 2016-03223).

\section{REFERENCES}

(1) Kojima, A.; Teshima, K.; Shirai, Y.; Miyasaka, T. Organometal Halide Perovskites as Visible-Light Sensitizers for Photovoltaic Cells. J. Am. Chem. Soc. 2009, 131, 6050-6051.

(2) Best Research-Cell Efficiencies. National Research and Energy Laboratroy, April 2020. https://www.nrel.gov/pv/assets/pdfs/bestresearch-cell-efficiencies.20200406.pdf.

(3) Park, N.-G. Research Direction toward Scalable, Stable, and High Efficiency Perovskite Solar Cells. Adv. Energy Mater. 2020, 10, 1903106.

(4) Green, M. A.; Ho-Baillie, A.; Snaith, H. J. The emergence of perovskite solar cells. Nat. Photonics 2014, 8, 506-514.

(5) Hao, F.; Stoumpos, C. C.; Chang, R. P.; Kanatzidis, M. G. Anomalous Band Gap Behavior in Mixed $\mathrm{Sn}$ and $\mathrm{Pb}$ Perovskites Enables Broadening of Absorption Spectrum in Solar Cells. J. Am. Chem. Soc. 2014, 136, 8094-8099.

(6) Pathak, S.; Sepe, A.; Sadhanala, A.; Deschler, F.; Haghighirad, A.; Sakai, N.; Goedel, K. C.; Stranks, S. D.; Noel, N.; Price, M.; Hüttner, S.; Hawkins, N. A.; Friend, R. H.; Steiner, U.; Snaith, H. J. Atmospheric Influence upon Crystallization and Electronic Disorder and Its Impact on the Photophysical Properties of Organic-Inorganic Perovskite Solar Cells. ACS Nano 2015, 9, 2311-2320.

(7) Hailegnaw, B.; Kirmayer, S.; Edri, E.; Hodes, G.; Cahen, D. Rain on Methylammonium Lead Iodide Based Perovskites: Possible Environmental Effects of Perovskite Solar Cells. J. Phys. Chem. Lett. 2015, 6, 1543-1547.

(8) Slavney, A. H.; Smaha, R. W.; Smith, I. C.; Jaffe, A.; Umeyama, D.; Karunadasa, H. I. Chemical Approaches to Addressing the Instability and Toxicity of Lead-Halide Perovskite Absorbers. Inorg. Chem. 2017, 56, 46-55.

(9) Noel, N. K.; Stranks, S. D.; Abate, A.; Wehrenfennig, C.; Guarnera, S.; Haghighirad, A.-A.; Sadhanala, A.; Eperon, G. E.; Pathak, S. K.; Johnston, M. B.; Petrozza, A.; Herz, L. M.; Snaith, H. J. Lead-free organic-inorganic tin halide perovskites for photovoltaic applications. Energy Environ. Sci. 2014, 7, 3061-3068.

(10) Leijtens, T.; Prasanna, R.; Gold-Parker, A.; Toney, M. F.; McGehee, M. D. Mechanism of tin oxidation and stabilization by lead substitution in tin halide perovskites. ACS Energy Lett. 2017, 2, 21592165.

(11) Ganose, A. M.; Savory, C. N.; Scanlon, D. O. Beyond methylammonium lead iodide: prospects for the emergent field of $\mathrm{ns}^{2}$ containing solar absorbers. Chem. Commun. 2017, 53, 20-44.

(12) Gao, X.; Zhang, X.; Yin, W.; Wang, H.; Hu, Y.; Zhang, Q.; Shi, Z.; Colvin, V. L.; Yu, W. W.; Zhang, Y. Ruddlesden-Popper Perovskites: Synthesis and Optical Properties for Optoelectronic Applications. Adv. Sci. 2019, 6, 1900941.

(13) Mitzi, D. B. Templating and structural engineering in organicinorganic perovskites. J. Chem. Soc., Dalton Trans. 2001, 0, 1-12.

(14) Katan, C.; Mercier, N.; Even, J. Quantum and Dielectric Confinement Effects in Lower-Dimensional Hybrid Perovskite Semiconductors. Chem. Rev. 2019, 119, 3140-3192.

(15) Liu, J.; Chen, K.; Khan, S. A.; Shabbir, B.; Zhang, Y.; Khan, Q.; Bao, Q. Synthesis and optical applications of low dimensional metalhalide Perovskites. Nanotechnology 2020, 31, 152002.

(16) Safdari, M.; Svensson, P. H.; Hoang, M. T.; Oh, I.; Kloo, L.; Gardner, J. M. Layered 2D alkyldiammonium lead iodide perovskites: synthesis, characterization, and use in solar cells. J. Mater. Chem. A 2016, 4, 15638-15646.

(17) Saparov, B.; Mitzi, D. B. Organic-Inorganic Perovskites: Structural Versatility for Functional Materials Design. Chem. Rev. 2016, 116, 4558-4596.
(18) Starkholm, A.; Kloo, L.; Svensson, P. H. Polyiodide hybrid perovskites: A strategy to convert intrinsic $2 \mathrm{D}$ systems into $3 \mathrm{D}$ photovoltaic materials. ACS Appl. Energy Mater. 2019, 2, 477-485.

(19) Evans, H. A.; Lehner, A. J.; Labram, J. G.; Fabini, D. H.; Barreda, O.; Smock, S. R.; Wu, G.; Chabinyc, M. L.; Seshadri, R.; Wudl, F. (TTF) $\mathrm{Pb}_{2} \mathrm{I}_{5}$ : A Radical Cation-Stabilized Hybrid Lead Iodide with Synergistic Optoelectronic Signatures. Chem. Mater. 2016, 28, 3607-3611.

(20) Mitzi, D. B.; Chondroudis, K.; Kagan, C. R. Design, Structure, and Optical Properties of Organic-Inorganic Perovskites Containing an Oligothiophene Chromophore. Inorg. Chem. 1999, 38, 6246-6256.

(21) Mitzi, D. B. Organic-Inorganic Perovskites Containing Trivalent Metal Halide Layers: The Templating Influence of the Organic Cation Layer. Inorg. Chem. 2000, 39, 6107-6113.

(22) Jana, M. K.; Janke, S. M.; Dirkes, D. J.; Dovletgeldi, S.; Liu, C.; Qin, X.; Gundogdu, K.; You, W.; Blum, V.; Mitzi, D. B. DirectBandgap 2D Silver-Bismuth Iodide Double Perovskite: The Structure-Directing Influence of an Oligothiophene Spacer Cation. J. Am. Chem. Soc. 2019, 141 (19), 7955-7964.

(23) Passarelli, J. V.; Fairfield, D. J.; Sather, N. A.; Hendricks, M. P.; Sai, H.; Stern, C. L.; Stupp, S. I. Enhanced Out-of-Plane Conductivity and Photovoltaic Performance in $\mathrm{n}=1$ Layered Perovskites through Organic Cation Design. J. Am. Chem. Soc. 2018, 140, 7313-7323.

(24) Febriansyah, B.; Koh, T. M.; John, R. A.; Ganguly, R.; Li, Y.; Bruno, A.; Mhaisalkar, S. G.; England, J. Inducing Panchromatic Absorption and Photoconductivity in Polycrystalline Molecular 1D Lead-Iodide Perovskites through $\pi$-Stacked Viologens. Chem. Mater. 2018, 30, 5827-5830.

(25) Liu, J.-J.; Guan, Y.-F.; Jiao, C.; Lin, M.-J.; Huang, C.-C.; Dai, W. A Panchromatic Hybrid Crystal of Iodoplumbate Nanowires and JAggregated Naphthalene Diimides with Long-Lived Charge-Separated States. Dalt. Trans. 2015, 44, 5957-5960.

(26) Marchal, N.; Van Gompel, W.; Gélvez-Rueda, M. C.; Vandewal, K.; Van Hecke, K.; Boyen, H.-G.; Conings, B.; Herckens, R.; Maheshwari, S.; Lutsen, L.; et al. Lead-Halide Perovskites Meet Donor-Acceptor Charge-Transfer Complexes. Chem. Mater. 2019, 31, 6880-6888.

(27) Chai, W.-X.; Lin, J.; Song, L.; Qin, L.-S.; Shi, H.-S.; Guo, J.-Y.; Shu, K.-Y. Three iodometalate organic-inorganic hybrid materials based on methylene blue cation: Syntheses, structures, properties and DFT calculations. Solid State Sci. 2012, 14, 1226-1232.

(28) Veron, A. C.; Linden, A.; Leclaire, N. A.; Roedern, E.; Hu, S.; Ren, W.; Rentsch, D.; Nuesch, F. A. One-Dimensional OrganicInorganic Hybrid Perovskite Incorporating Near-Infrared-Absorbing Cyanine Cations. J. Phys. Chem. Lett. 2018, 9, 2438-2442.

(29) Saccone, D.; Galliano, S.; Barbero, N.; Quagliotto, P.; Viscardi, G.; Barolo, C. Polymethine Dyes in Hybrid Photovoltaics: StructureProperties Relationships. Eur. J. Org. Chem. 2016, 2016, 2244-2259.

(30) Chen, S.; Hou, Y.; Chen, H.; Tang, X.; Langner, S.; Li, N.; Stubhan, T.; Levchuk, I.; Gu, E.; Osvet, A.; Brabec, C. J. Exploring the Stability of Novel Wide Bandgap Perovskites by a Robot based High throughput Screening Approach. Adv. Energy Mater. 2018, 8, 1701543-1701550.

(31) Sun, S.; Hartono, N. T. P.; Ren, Z. D.; Oviedo, F.; Buscemi, A. M.; Layurova, M.; Chen, D. X.; Ogunfunmi, T.; Thapa, J.; Ramasamy, S.; Settens, C.; DeCost, B. L.; Kusne, A. G.; Liu, Z.; Tian, S. I. P.; Peters, I. M.; Correa-Baena, J.-P.; Buonassisi, T. Accelerated Development of Perovskite-Inspired Materials via High-Throughput Synthesis and Machine-Learning Diagnosis. Joule. 2019, 3, 14371451.

(32) Würthner, F.; Kaiser, T. E.; Saha-Möller, C. R. J-Aggregates: From Serendipitous Discovery to Supra-molecular Engineering of Functional Dye Materials. Angew. Chem., Int. Ed. 2011, 50, 33763410.

(33) Hagfeldt, A.; Boschloo, G.; Sun, L.; Kloo, L.; Pettersson, H. Dye-Sensitized Solar Cells. Chem. Rev. 2010, 110, 6595-6663.

(34) Kubelka, P.; Munk, F. Ein Beitrag Zur Optik Der Farbanstriche. Z. Technol. Phys. 1931, 12, 593-601. 
(35) Xu, B.; Jiao, K.; Sun, W.; Zhang, X. Recognition and Determination of DNA Using Victoria Blue B as Electrochemical Probe. Int. J. Electrochem. Sci. 2007, 2, 406-417.

(36) Papavassiliou, G. C.; Koutselas, I. B. Structural, Optical and Related Properties of some Natural Three- and Lower-Dimensional Semiconductor Systems. Synth. Met. 1995, 71, 1713-1714.

(37) Muruganandham, M.; Swaminathan, M. Photochemical oxidation of reactive azo dye with $\mathrm{UV}-\mathrm{H}_{2} \mathrm{O}_{2}$ process. Dyes Pigm. 2004, 62, 269-275.

(38) Mosconi, E.; Umari, P.; De Angelis, F. Electronic and optical properties of $\mathrm{MAPbX}_{3}$ perovskites $(\mathrm{X}=\mathrm{I}, \mathrm{Br}, \mathrm{Cl})$ : a unified DFT and GW theoretical analysis. Phys. Chem. Chem. Phys. 2016, 18, 2715827164 .

(39) Singh, R.; Sandhu, S.; Lee, J.-J. Elucidating the effect of shunt losses on the performance of mesoporous perovskite solar cells. Sol. Energy 2019, 193, 956-961.

(40) Aranda, C.; Bisquert, J.; Guerrero, A. Impedance spectroscopy of perovskite/contact Interface: Beneficial chemical reactivity effect. $J$. Chem. Phys. 2019, 151, 124201-124209.

(41) Abate, A.; Leijtens, T.; Pathak, S.; Teuscher, J.; Avolio, R.; Errico, M. E.; Kirkpatrik, J.; Ball, J. M.; Docampo, P.; McPherson, I.; Snaith, H. J. Lithium salts as "redox active" p-type dopants for organic semiconductors and their impact in solid-state dye-sensitized solar cells. Phys. Chem. Chem. Phys. 2013, 15, 2572-2579.

(42) Snaith, H. J.; Grätzel, M. Enhanced charge mobility in a molecular hole transporter via addition of redox inactive ionic dopant: Implication to dye-sensitized solar cells. Appl. Phys. Lett. 2006, 89, 262114.

(43) Noh, J.; Jeon, N.; Choi, Y.; Grätzel, M.; Nazeeruddin, M.; Seok, S. Nanostructured $\mathrm{TiO}_{2} / \mathrm{CH}_{3} \mathrm{NH}_{3} \mathrm{PbI}_{3}$ heterojunction solar cells employing spiro-OMeTAD/Co-complex as hole-transporting material. J. Mater. Chem. A 2013, 1, 11842-11847.

(44) Schölin, R.; Karlsson, M. H.; Eriksson, S. K.; Siegbahn, H.; Johansson, E. M. J.; Rensmo, H. Energy Level Shifts in SpiroOMeTAD Molecular Thin Films When Adding Li-TFSI. J. Phys. Chem. C 2012, 116, 26300-26305.

(45) Zhao, Y.; Zhu, K. Charge Transport and Recombination in Perovskite $\left(\mathrm{CH}_{3} \mathrm{NH}_{3}\right) \mathrm{PbI}_{3}$ Sensitized $\mathrm{TiO}_{2}$ Solar Cells. J. Phys. Chem. Lett. 2013, 4, 2880-2884.

(46) Liu, D.; Gangishetty, M. K.; Kelly, T. L. Effect of $\mathrm{CH}_{3} \mathrm{NH}_{3} \mathrm{PbI}_{3}$ thickness on device efficiency in planar heterojunction perovskite solar cells. J. Mater. Chem. A 2014, 2, 19873-19881.

(47) Safdari, M.; Phuyal, D.; Philippe, B.; Svensson, P. H.; Butorin, S. M.; Kvashnina, K. O.; Rensmo, H.; Kloo, L.; Gardner, J. M. Impact of synthetic routes on the structural and physical properties of butyl1,4-diammonium lead iodide semiconductors. J. Mater. Chem. A 2017, 5, 11730-11738.

(48) Adonin, S. A.; Frolova, L. A.; Sokolov, M. N.; Shilov, G. V.; Korchagin, D. V.; Fedin, V. P.; Aldoshin, S. M.; Stevenson, K. J.; Troshin, P. A. Antimony (V) Complex Halides: Lead-Free PerovskiteLike Materials for Hybrid Solar Cells. Adv. Energy Mater. 2018, 8, 1701140-1701146.

(49) Chen, M.; Ju, M.-G.; Carl, A. D.; Zong, Y.; Grimm, R. L.; Gu, J.; Zeng, X. C.; Zhou, Y.; Padture, N. P. Cesium titanium(IV) bromide thin films based stable lead-free perovskite solar cells. Joule 2018, 2, 558-570.

(50) Bruker Apex II, 2013.2-0; Bruker AXS: Madison, WI, 2013.

(51) Dovesi, R.; Erba, A.; Orlando, R.; Zicovich-Wilson, C. M.; Civalleri, B.; Maschio, L.; Rerat, M.; Casassa, S.; Baima, J.; Salustro, S.; Kirtman, B. Quantum-mechanical condensed matter simulations with CRYSTAL. WIREs Comp. Mol. Sci. 2018, 8, e1360. Crystal 17.

(52) Stoll, H.; Metz, B.; Dolg, M. J. J. Comput. Chem. 2002, 23, 767-778.

(53) Metz, B.; Stoll, H.; Dolg, M. Small-core multiconfigurationDirac-Hartree-Fock-adjusted pseudopotentials for post-d main group elements: Application to $\mathrm{PbH}$ and $\mathrm{PbO}$. J. Chem. Phys. 2000, $113,2563-2569$.
(54) Peterson, K. A.; Shepler, B. C.; Figgen, D.; Stoll, H. On the Spectroscopic and Thermochemical Properties of $\mathrm{ClO}, \mathrm{BrO}, \mathrm{IO}$, and Their Anions. J. Phys. Chem. A 2006, 110, 13877-13883.

(55) Peterson, K. A. Systematically convergent basis sets with relativistic pseudopotentials. I. Correlation consistent basis sets for the post-d group 13-15 elements. J. Chem. Phys. 2003, 119, 1109911112.

(56) Bergner, A.; Dolg, M.; Küchle, W.; Stoll, H.; Preuss, H. Ab initio energy-adjusted pseudopotentials for elements of groups 13-17. Mol. Phys. 1993, 80, 1431-1441.

(57) http://crysplot.crystalsolutions.eu/, October 2019.

(58) Frisch, M. J.; Trucks, G. W.; Schlegel, H. B.; Scuseria, G. E.; Robb, M. A.; Cheeseman, J. R.; Scalmani, G.; Barone, V.; Mennucci, B.; Petersson, G. A.; Nakatsuji, H.; Caricato, M.; Li, X.; Hratchian, H. P.; Izmaylov, A. F.; Bloino, J.; Zheng, G.; Sonnenberg, J. L.; Hada, M.; Ehara, M.; Toyota, K.; Fukuda, R.; Hasegawa, J.; Ishida, M.; Nakajima, T.; Honda, Y.; Kitao, O.; Nakai, H.; Vreven, T.; Montgomery, J. A., Jr.; Peralta, J. E.; Ogliaro, F.; Bearpark, M.; Heyd, J. J.; Brothers, E.; Kudin, K. N.; Staroverov, V. N.; Kobayashi, R.; Normand, J.; Raghavachari, K.; Rendell, A.; Burant, J. C.; Iyengar, S. S.; Tomasi, J.; Cossi, M.; Rega, N.; Millam, J. M.; Klene, M.; Knox, J. E.; Cross, J. B.; Bakken, V.; Adamo, C.; Jaramillo, J.; Gomperts, R.; Stratmann, R. E.; Yazyev, O.; Austin, A. J.; Cammi, R.; Pomelli, C.; Ochterski, J. W.; Martin, R. L.; Morokuma, K.; Zakrzewski, V. G.; Voth, G. A.; Salvador, P.; Dannenberg, J. J.; Dapprich, S.; Daniels, A. D.; Farkas, O.; Foresman, J. B.; Ortiz, J. V.; Cioslowski, J.; Fox, D. J. Gaussian 16, Rev. B.01; Gaussian, Inc.: Wallingford, CT, 2016. 